\title{
A novel 2-stage dish concentrator with improved optical performance for concentrating solar power plants
}

\author{
Jun Wang ${ }^{1}$, Song Yang ${ }^{1}$, Chuan Jiang ${ }^{1}$, Qianwen Yan ${ }^{1}$, Peter D. Lund ${ }^{1,2}$
}

\author{
${ }^{1}$ Key Laboratory of Solar Energy Science and Technology in Jiangsu Province \\ Southeast University, Solar Energy Center, No.2 Si Pai Lou, Nanjing 210096, China \\ ${ }^{2}$ Aalto University School of Science, P.O. Box 15100, FI-00076 Aalto (Espoo), Finland
}

\begin{abstract}
Here we propose a novel 2-stage dish concentrator system for concentrating solar power plants based on the so-called overlap method, which improves both the radial flux concentration ratio $(\beta)$ and the intercept factor $(\gamma)$ of the concentrator. The 2-stage dish is composed of paraboloid primary and hyperboloid secondary mirrors having a special hollow structure enabling the mirrors to overlap, but avoiding excess shading. Geometric and optical analyses with ray-tracing show maximum values of $\beta=15,498$ and $\gamma=0.78$. Also, the size of the focal spot could be reduced. The concept is applicable for solar tower concepts enabling more compact unit mirrors and reducing the receiver size.
\end{abstract}

Key words: CSP; solar dish; solar tower; overlap method; ray-tracing 


\section{Nomenclature}

Symbols

C Geometrical concentration radio

D Diameter of PM

d Diameter of SM

$\mathrm{e}_{\mathrm{x}} \quad$ Unit vector in $\mathrm{x}$-axis direction

$\frac{\mathrm{f}}{I_{a}} \quad$ Focal length

Average irradiance absorbed on receiver

$\mathrm{I}_{0} \quad$ Incident irradiance

NA Sinus of half field angle between mirror edge and central axis

$\mathrm{r}_{\mathrm{a}} \quad$ Radius of concentrated spot

$\theta \quad$ Half edge angle of mirror 1

$\varphi \quad$ Half field angle between edge of mirror 2 and $\mathrm{x}$-axis

$\alpha \quad$ Shading losses

$\beta \quad$ Radial flux concentration radio

$\gamma \quad$ Intercept factor

Subscripts

$1 \quad$ PM (Mirror 1)

$2 \quad$ SM (Mirror 2)

$3 \quad \mathrm{TM}$

$\mathrm{p} \quad \mathrm{PM}$ (Mirror 1) 
Abbreviations

CPC Compound parabolic concentrator

CSP Concentrating solar power

EFMGTExternally fired motor gas turbine

IFMGT Internally fired motor gas turbine

PM Primary mirror (Mirror 1)

SM Secondary mirror (Mirror 2)

SM1 Secondary hyperboloid mirror

SM2 Secondary paraboloid mirror

TES Thermal energy storage

TM Tertiary mirror

\section{Introduction}

Concentrating solar power (CSP) is a promising renewable power technology for regions with high solar radiation intensity [1]. In a recent scenario by the International Energy Agency, the share of CSP in global electricity production could reach $11 \%$ by 2050 [2]. Though CSP has several advantages such as hybridization and dispatchability, the main challenge is the high cost as CSP is not yet cost-competitive with conventional fossil fuel technologies [3].

Commercial CSP plants are typically based on single-axis tracking parabolic trough systems employing a Rankine steam cycle [4]. The concentration ratio (C) of such 2-dimensional concentrators is limited to a few hundred Suns, which in turn limits the maximum achievable temperature and the plant conversion efficiency to around $20 \%$. Increasing the temperature would require 3-dimensional parabolic dishes with 2-axis solar tracking, which could reach 
C 1,000. Such systems have been realized as point-focused in combination with small Stirling-engines reaching solar-to-power conversion efficiencies beyond $30 \%$, even closer to 40\% [5]. However, a larger CSP system based on the Stirling cycle would require a very large amount of small units due to limited engine power (10-40kW) [6] and dish size, thus often limiting the applicability of Dish-Stirling engines to remote areas with small power demands and higher energy costs. Solar power tower systems reach similar concentration ratios, but with a large heliostat field. The land requirement of a large plant with thermal energy storage (TES) is about 4 ha per $\mathrm{MW}_{\mathrm{e}}$ [7]. The tower concept often employs a steam cycle, but due to potentially higher temperatures, it could also run on a Brayton gas cycle. Demonstration projects of this type include e.g. the Weizmann Solar Tower (Israel), SOLGATE and SOLHYCO (Spain)[8], and Jianging Solar Tower (China)[9].

Developing improved solar concentrator-power cycle concepts is highly motivated for better efficiency and for lowering the cost of CSP. Also, the Brayton cycle needs less water than the Rankine cycle. Therefore, an interesting approach could be to combine parabolic dish concentrators for a solar power tower with a Brayton cycle. The idea is to employ a large dish instead of planar heliostat mirrors for a higher concentration ratio, even at smaller scale. As this concept would inherently lead to large dishes, we propose an improved two-stage dish concentrator, which is more compact and flexible and has both a very high concentration ratio and a good intercept factor. This would potentially require less land area than a tower plant of the same scale. Another motivation of the study was to find a more effective mirror system for the Jianging solar tower system in Nanjing China, which employs a Brayton power cycle with a central receiver and heliostat system [9]. The literature on solar Brayton cycles is ample covering a broad range of topics from basic thermal engineering [11-15] to receiver and heat exchanger design $[16,17]$.

The main focus of this paper is on the optical design of the new dish system. This includes designing a novel two-stage dish concentrator applicable to the tower concept (but not bound to this), but also introducing an improved method for increasing the concentration ratio and intercept factor of the 2-stage collector employing the focusing overlap method.

2-stage concentrators impose a basic design challenge, because it is difficult simultaneously to 
reach a high concentration ratio and a good intercept factor. This is because the shadowing of the secondary mirrors grows when increasing the mirror size, which drops the intercept factor. Goldstein et al proposed a new structure for concentrating PV, the so-called Nested aplanats, to maximize the solar concentrator performance, e.g. using two Cassegrainian mirrors [10]. They introduced a special hollow and overlap dish collector for the first time, but lacked more detailed analyses. Moreover, their algorithm to deduce geometric structures of key components was quite complex, which we try to overcome here. Based on the nested combination, this paper introduces the overlap focusing method and some further improvements, including simplified geometric formulas, building a novel prototype, presenting accurate ray tracing analysis, and a range of quantitative analyses of key factors. We demonstrate here a clear improvement through the new dish design. The overlap focusing method proposed could enable both a high concentration ratio and a good intercept factor. Also, it helps to lower the level of radiation fluxes on the secondary reflectors thus reducing the surface temperatures on these mirrors.

\section{Optical modelling of 2-stage concentrator}

To reach a high concentration ratio above 1,0000, a paraboloidal dish would be practical. Moreover, a two-stage concentrator could provide better flexibility and a stable structure. Based on the compound parabolic concentrator (CPC) principle [18], Rabl et al [19] tested a combination of CPC as a second stage concentrator for a conventional parabolic or Fresnel mirror, demonstrating significant advantages. A Cassegrainian type of concept (parabolic \& hyperbolic) was investigated by Mauk et al [20] for solar energy use in a chemical system. Zhang et al [21] discussed five different types of two-stages reflectors based on a primary parabolic mirror. Mora and Jaramillo [22] used porous silicon photonic mirrors as secondary reflectors. Actually, five different types of secondary mirrors can be identified: flat mirrors [23], ellipsoidal mirrors [24], hyperboloidal mirrors with upper sheet [25] and lower sheet [26], and paraboloidal mirrors [27]. The concentrator system considered here is presented in the next.

2.1. Geometric model of the concentrator 
Figure 1 illustrates the optical system of interest, which consists of two main dish mirrors (mirror 1 and 2). We denote this concept as the reference concentrator system. The sun-light reflects from mirror 1 toward its focal point in front of which we have mirror 2 from which the lights reflects back to the front focal point of mirror 2, where the receiver is placed.

For mathematical modeling, a combination of a paraboloid (PM) and hyperboloid (SM) mirror is selected as shown in Fig.1. The mirrors are curved and placed in such a way that the focal lengths are the same $\left(f_{1}=f_{2}\right)$. Let's denote the mirror diameters with $D$ and $d ; \theta / \varphi$ is the half field angle between the edge of mirror 1 or mirror 2 and the central axis. The curvatures of the mirrors are then given by the following geometric equations, where subscript 1 refers to PM (1) and 2 to $\mathrm{SM}(2)$ :

$$
\begin{aligned}
& y_{1}= \pm 4 f_{1} x_{1} \\
& \mathrm{y}_{2}= \pm \sqrt{\frac{b^{2}}{a^{2}}\left(x_{2}-c\right)^{2}-b^{2}}
\end{aligned}
$$

Denoting $\mathrm{NA}_{1}=\sin \theta_{1}, \mathrm{NA}_{2}=\sin \theta_{2}, \theta_{1}=\varphi$, and $\theta_{2}=\theta$, the following set of system equations can be derived:

$$
\begin{aligned}
& \tan \varphi=\frac{8}{16\left(f_{1} / D\right)-\left(d / f_{1}\right)} \\
& m=\frac{\overrightarrow{O F_{2}} \cdot e_{x}}{f_{1}} \\
& d=\frac{4 c}{1 / \tan \theta+1 / \tan \varphi}
\end{aligned}
$$




$$
b=\sqrt{c^{2}-a^{2}}
$$

$$
\begin{aligned}
& a=\frac{D}{4} \cdot\left(\frac{1}{\sin \theta}-\frac{1}{\sin \varphi}\right) \\
& c=f_{2} / 2=\left(f_{1}-m f_{1}\right) / 2
\end{aligned}
$$

$\mathrm{e}_{\mathrm{x}}$ is a unit vector in $\mathrm{x}$-axis direction, $\overrightarrow{\mathrm{OF}}_{2}$ defines the vector from the receiver to the left focal point of mirror 2. Setting $\mathrm{m}$ to zero simplifies the solution. To analyze the relationships between the optical effects and geometrical factors $\left(\mathrm{NA}_{2} / \mathrm{f}_{1}\right)$, we use one of these as a variable and keep the other constant, or vice versa. For example, if $f_{1}$ is preset at $10 \mathrm{~m}$, then $\mathrm{NA}_{2}$ is gradually varied from 0.1 to 0.9 .

\subsection{Ray-trace simulations}

Ray tracing simulation is a very effective tool to design and optimize parameters of solar concentrators [28]. For the optical analysis, we employed the Tracepro ray-tracing tool [34]. In the analysis, we fixed mirror 1 and varied mirror 2 . The assumptions for the simulation were as follows:

- The incident irradiance $\left(\mathrm{I}_{0}\right)$ is $996 \mathrm{~W} / \mathrm{m}^{2}$. All incident light is sunlight with a half angle of 16 '[29];

- The diameter of mirror 1 is $20 \mathrm{~m}$, reflectivity is 0.949 , and the receiver/absorber is a black body; 
- The effects of other random and non-random errors are neglected (e.g. random slope errors, and reflector profile imperfection, misalignment errors, receiver location errors, etc.)[30].

Moreover, we make the following definitions:

- The radius of the concentrated spot $\left(=r_{a}\right)$ is equal to the radius of the maximal circle on the edge of which radiant flux densities have decayed to $1 / \mathrm{e}^{2}$ of the peaked value [31];

- Both the geometrical $(\mathrm{C})$ and radial $(\beta)$ concentration ratio are considered:

$$
\begin{gathered}
\mathrm{C}=\frac{D^{2}}{4 r_{a}^{2}} \\
\beta=\frac{\overline{I_{a}}}{I_{0}}
\end{gathered}
$$

$\overline{I_{a}}$ is the average of the radiant flux density absorbed on the flat receiver.

- The intercept factor of the concentrator system $(\gamma)$ is defined as the fraction (0-1) of rays incident upon the aperture that reach the receiver [32];

- In a 2-stage solar concentrator system, shading losses $(\alpha)$ may contribute to a low intercept factor:

$$
\alpha=\frac{\mathrm{A}_{\mathrm{s}}}{\mathrm{A}_{\mathrm{p}}}
$$

$\mathrm{A}=$ area; subscript $\mathrm{p}$ denotes the Primary Mirror $(\mathrm{PM} ; 1)$ and s the Secondary Mirror (SM; 2).

\subsection{Results of ray-trace analysis}

Using the optical geometry defined in Chapter 2.1 and the assumptions from Chapter 2.2, we performed a ray-trace analysis of the reference concentrator system in Fig. 1 to estimate the concentration factors for different geometries.

Figure 2 (a) shows the geometrical concentration ratio (C) as a function of the half field angle 
of SM. The PM's focal length $\left(\mathrm{f}_{1}\right)$ is set to $5,000 \mathrm{~mm}, 10,000 \mathrm{~mm}$, and $15,000 \mathrm{~mm}$; $\mathrm{NA}_{2}$ varies from 0.1 to 0.85 . The concentration ratios in Fig. 2 remain almost the same up to $\mathrm{NA}_{2} \sim 0.45$ meaning that the impact from $\mathrm{f}_{1}$ to $\mathrm{C}$ is negligible in this region. But with higher $\mathrm{NA}_{2}$ values, the curves start to separate till reaching a maximum value meaning that the PM's structure starts to influence the final results. In addition, looking at the tail end of the curve for $\mathrm{f}_{1}=5,000 \mathrm{~mm}$, we can see a rapid decline after reaching a maximum value. It is due to the effect of the solar half angle, which limits the minimum focal spot size. If $\mathrm{NA}_{2}>0.75$ and $\mathrm{NA}_{2}$ keeps increasing, the diameter of the spot cannot reduce anymore, but may start to expand. The largest apertures of the spots relative to the lowest $\mathrm{C}$ appear when $\mathrm{NA}_{2}$ is at minimum. For instance, $\mathrm{C}$ is 445,457 , and 451 for the three values of $\mathrm{f}_{1}$ when $\mathrm{NA}_{2}=0.1$. As $\theta$ expands, $\mathrm{C}$ grows and in the middle region the curves are close to linear. However, the growth of $\mathrm{C}$ is possible only up to a limited value [33] and the slope of the curve starts to decline at a high $\theta$ $\left(\mathrm{NA}_{2}>0.7\right)$ as explained above. A maximum value of $\mathrm{C}=20,408\left(\mathrm{f}_{1}=10000 \mathrm{~mm}\right)$ was found at $\mathrm{NA}_{2}=0.79$. The data range of $\mathrm{NA}_{2}$ in Fig. 2 is from 0 to $0.9,0$ to 0.8 , and 0 to 0.6 for $\mathrm{f}_{1}=5,000 \mathrm{~mm}, 10,000 \mathrm{~mm}$ and $15,000 \mathrm{~mm}$, correspondingly.

Figure $2(\mathrm{~b})$ shows the changes in the radial flux concentration ratio $(\beta)$ when varying $\mathrm{NA}_{2}$. The behaviour of $\beta$ differs from that of $\mathrm{C}$. When $\mathrm{NA}_{2}<0.3$, the three curves show similarity. In the region $\mathrm{NA}_{2}>0.3$ when $\theta$ increases, shading losses turn out to be more serious, resulting in different shapes of the $\beta-\mathrm{NA}_{2}$ and $\mathrm{C}-\mathrm{NA}_{2}$ curves.

From Figure 2(b), $\beta$ could reach 11,792 $\left(\mathrm{f}_{1}=5,000 \mathrm{~mm}\right)$ with $\mathrm{NA}_{2}=0.65-0.75$, which is slightly higher than 10,749 with $\mathrm{f}_{1}=10,000 \mathrm{~mm}$ for the same range of $\mathrm{NA}_{2}$. For $\mathrm{f}_{1}=15,000 \mathrm{~mm}$, the maximum value of $\beta$ is $8,408\left(\mathrm{NA}_{2} \sim 0.6\right)$.

For the optical design of concentrators, $\beta$ is a more useful parameter than $\mathrm{C}$. The optimized concentrator design parameters for this reference case would then be $\mathrm{NA}_{2}=0.7$ and $\mathrm{f}_{1}=5,000 \mathrm{~mm}$.

\section{Focusing overlap method}


Though a high concentration ratio $(\beta)$ was obtained with the reference system, a high intercept factor $(\gamma)$ could not be attained, but it only reached a value of 0.663 , i.e. one third of the incident flux was lost. Most of the losses were due to shadows from the secondary mirror (SM). Figure 3 shows how $\beta$ and $\gamma$ change with $\mathrm{NA}_{2}$, which well demonstrates that with the reference concentrator design it would not be possible to simultaneously reach a high $\beta$ and high $\gamma$.

As a solution for this 'paradox', we introduce an improved design approach called the focusing overlap method, which could enable both a high concentration ratio and high intercept factor. The structure of the improve concept is described in the next.

Figure 4 illustrates the prototype of a modified two-stage concentrator based on the focusing overlap method, which we here call the novel design. Compared to the reference concentrator system, in which only two mirrors (PM and SM) are used, we propose four tailored mirrors. Looking in from outside, the mirror units are: 1) primary mirror (PM1), 2) front secondary mirror (SM1), 3) back secondary mirror (SM2) and 4) tertiary mirror (TM), successively.

PM and SM1 are similar with the primary and secondary mirrors in the reference design, constituting of a paraboloid and a hyperboloid, but are here cut into annular forms. This design reduces the mirror cost and alleviates structural loads. The shaded zoom in PM is equal to the projected area of SM1, which is useless in the unit and could thus be eliminated. The hollowed structure of SM1 was planned so that the rays reflected from the TM could get through it. The region around the hole in SM1 is limited by two factors: rays from the PM should get reflected to the left surface of SM1, and light concentrated by the TM should penetrate the hole.

As shown in Fig.4, another two-stage reflector, but smaller in size, was furnished close to the former one. The new PM, denoted as SM2, was used to intercept the rest of the incident rays, shielded by SM1, and it reflects them into the next mirror TM. Through this additional step, a part of the radiation could be utilized and then focused on the receiver. By overlapping the two 2-stage reflectors, the total radial flux and radiation densities could intuitively be improved. To verify this, we modified the optical calculations with the overlapping design. Table 1 gives the main parameters of the new prototype. $\mathrm{NA}_{3}$ was defined as sinus of the half field angle between TM's edge and the central axis (range 0.1-0.6). 
Figures 5 and 6 show how $\beta, \gamma$, and $\alpha$ change with $\mathrm{NA}_{3}(0.1-0.6)$. In this case, the size and shape of PM and SM1 are both chosen $\left(\mathrm{f}_{1}=5,000 \mathrm{~mm}, \mathrm{NA}_{2}=0.79\right)$ to yield the highest $\mathrm{C}$ and a compact structure.

In the $\mathrm{NA}_{3}-\beta$ diagram, line 1 corresponds to the additional concentration effect by $\mathrm{SM}_{2}$ and $\mathrm{TM}$. When increasing $\mathrm{NA}_{3}, \beta$ increases up to a peak value and then gradually starts to drop. The highest $\beta$ equal to 15,498 was obtained when $\mathrm{NA}_{3}$ is close to 0.42 . Line 2 does not include these effects and the value of $\beta$ is therefore constant $(\beta=9,680)$. The concentration enhancement in Fig. 5 (line 1 vis-à-vis line 2) is significant, but the difference varies with $\mathrm{NA}_{3}$. When being above $\mathrm{NA}_{3}=0.6$, the size of SM1's hole cannot ensure that sun's rays from both sides could completely pass through. Consequently, a part of the sunlight will be shaded or cannot be reflected, which leads to a sharp decay in $\beta$ when $\mathrm{NA}_{3}>0.6$.

In Fig.6, $\gamma$ of the new design reaches a maximum at $\mathrm{NA}_{3}=0.4-0.45$, i.e. in the same range where $\beta$ reaches its maximum. Parameter $\alpha$ is a monotonously increasing function of $\mathrm{NA}_{3 . .} \gamma$ and $\alpha$ remain constant when the effects from SM2 and TM are excluded. Due to shading losses, the optical performance is much lower $(\gamma=0.502)$ in this case. With the new design it is possible to reach $\beta>14,000$ and $\gamma>0.7$ with $\mathrm{NA}_{3}=0.35-0.5$.

Finally, we made race-trace stimulations with TracePro [34] and compared the new and reference concentrator designs in Fig.7, which shows the irradiance distribution in the focal as a gradient map and curve diagram. The radius of the spot is $75 \mathrm{~mm}$. For the reference design in Fig. 7 (a), the maximum irradiance within the circle area is $22.74 \mathrm{MW} / \mathrm{m}^{2}$ and the average is 11.8 MW/m². The total flux received by the receiver is $207.6 \mathrm{~kW}$. Figure $7(\mathrm{~b})$ shows the result for the new design by the overlap method. The radius of the spot is $71 \mathrm{~mm}$. The maximum and the average irradiance values are $28.6 \mathrm{MW} / \mathrm{m}^{2}(+25.8 \%)$ and $15.4 \mathrm{MW} / \mathrm{m}^{2}(+32.5 \%)$, respectively. The total flux is $244.5 \mathrm{~kW}(+18 \%)$. These values clearly demonstrate the enhanced performance of the new design.

\section{Conclusions}

A new 2-stage concentrator design based on the overlap method was described. It consists of a 
primary mirror (PM), two secondary mirrors (SM1 and SM2) and a tertiary mirror (TM). For comparison, we used a 2-stage concentrator consisting of a primary (PM) and secondary mirror (SM) as the reference case.

We showed using comprehensive optical analyses and ray-trace simulations that the new design with four tailored mirrors of which two slightly overlapped, demonstrated a clear performance improvement compared to the reference concentrator system with two mirrors. Both the concentration ratio $(\beta)$ and the intercept factor $(\gamma)$ could simultaneously be improved.

The two-stage dish reference system with a short focal length of $\mathrm{f}_{1}=5,000 \mathrm{~mm}$ yielded a peak concentration value of $\beta=11,792\left(\mathrm{NA}_{2}=0.7\right)$ and an intercept factor $\gamma=0.663$.

The improved concentrator based on the overlap design gave $\beta=15,498$ and $\gamma=0.781$ with $\mathrm{f}_{1}=5,000 \mathrm{~mm}, \mathrm{NA}_{2}=0.79$, and $\mathrm{NA}_{3}=0.42$. This means that $\beta$ was increased by $31.4 \%$ and $\gamma$ by $17.8 \%$. Also, the focal spot shrunk by $11.6 \%$ meaning that the aperture of the receiver could be reduced, which in turn would reduce the heat losses.

Summarizing, a 2-stage concentrator with four mirrors employing the overlap method yields a clearly better optical performance than a 2-stage concentrator with two mirrors, which could be beneficial for finding more cost-effective CSP solutions.

\section{Acknowledgements}

This work was supported by the National Science Foundation of China (No. 51476099).

\section{References}

[1] Z. Yao, Z. Wang, Z. Lu, X. Wei, Modeling and simulation of the pioneer 1MW solar thermal central receiver system in China, Renewable Energy 34 (2009) 2437-2446.

[2] C. Philiber, Technology Roadmap: Solar Thermal Electricity - 2014 edition. Renewable Energy Division, International Energy Agency, Paris, 2014.

[3] Annual Energy Outlook 2014. U.S. Energy Information Administration, 
Washington, DC, 2014.

[4] H. Müller-Steinhagen, F. Trieb, Concentrating solar power - a review of the technology, Ingenia 18(2004) 43-50.

[5] D. Mills, Advances in solar thermal electricity technology, Solar Energy 76 (2004) 19-31.

[6] Y. Li, S. Liao, Z. Rao, G. Liu, A dynamic assessment based feasibility study of concentrating solar power in China, Renewable Energy 69 (2014) 34-42.

[7] D. Kearney, Utility-scale power tower solar systems: performance acceptance test guidelines, Energy Procedia 49 (2014)1784-1791.

[8] B. Anderson, Brayton-cycle baseload power tower CSP system, Wilson Solar power in US Department of Energy (2013).

[9] J. Wang, Y. Zhang, D Liu, Introduction of the First Solar Power Tower System in China. Proceedings of ISES World Congress (Vol. I - Vol. V). Springer Berlin Heidelberg, (2007) 1738-1742.

[10] A. Goldstein, D. Feuermann, G. D. Conley, J. M. Gordon, Nested aplanats for practical maximum-performance solar concentration, Optics Letters 36 (2011) 2836-8.

[11] K. Bammert, H. Lange, Part-load behavior of a solar-heated and fossil-fueled gas turbine power plant, Journal of Engineering for Gas Turbines \& Power 10 (1987) 64-70.

[12] A. Pietsch, D. J. Brandes, Advanced solar Brayton space power systems. Energy Conversion Engineering Conference, Iecec-89. Proceedings of the Intersociety (Vol.2) IEEE. (1989) 911-916.

[13] B. Dickey, Test Results From a Concentrated Solar Microturbine Brayton Cycle Integration. ASME 2011 Turbo Expo: Turbine Technical Conference and Exposition (2011) 1031-1036 .

[14] W. Wang, G. Ragnolo, L. Aichmayer, T. Strand, B. Laumert, Integrated design of a hybrid gas turbine-receiver unit for a solar dish system, Energy Procedia, 69 (2015) 583-592. 
[15] L. Aichmayer, J. Spelling, B. Laumert, T. Fransson, Micro gas-turbine design for small-scale hybrid solar power plants, Journal of Engineering for Gas Turbines \& Power, 135 (2013) V004T05A002.

[16] J. Karni, A. Kribus, R. Rubin, P. Doron, The "porcupine": a novel high-flux absorber for volumetric solar receivers, Journal of Solar Energy Engineering, 120 (1998) 85-95.

[17] P. Poživil, V. Aga, A. Zagorskiy, A. Steinfeld, A pressurized air receiver for solar-driven gas turbines, Energy Procedia 49 (2014) 498-503.

[18] C. K. Hsieh, Thermal analysis of CPC collectors, Solar Energy 27 (1981) 19-29.

[19] A. Rabl, Comparison of solar concentrators, Solar Energy 18 (1976) 93-111.

[20] C. E. Mauk, H.W. Prengle, C. H. Sun, Optical and thermal analysis of a Cassegrainian solar concentrator, Solar Energy 23 (1979) 157-167.

[21] Y. Zhang, G. Xiao, Z. Luo, M. Ni, T. Yang, W. Xu, Comparison of different types of secondary mirrors for solar application. Optik - International Journal for Light and Electron Optics, 125 (2014) 1106-1112.

[22] M. B. D. L. Mora, O. A. Jaramillo, R. Nava, J. A. D. Río, Viability study of porous silicon photonic mirrors as secondary reflectors for solar concentration systems, Solar Energy Materials \& Solar Cells, 93(2009) 1218-1224.

[23] A. D. Feuermann, J. M. Gordon, High-concentration photovoltaic designs based on miniature parabolic dishes, Proceedings of SPIE - The International Society for Optical Engineering 70 (2001) 423-430.

[24] A. Segal, M. Epstein, The optics of the solar tower reflector. Solar Energy 69 (2001), 229-241.

[25] A. Kribus, R. Zaibel, A. Segal, Extension of the hermite expansion method for Cassegrainian solar central receiver systems. Solar Energy 63 (1998) 337-343.

[26] D. Feuermann, J. M. Gordon, H. Ries, High-flux solar concentration with imaging designs, Solar Energy 65 (1999) 83-89.

[27] S. Jiang, P. Hu, S. Mo, Z. Chen, Optical modeling for a two-stage parabolic trough concentrating photovoltaic/thermal system using spectral beam splitting technology, Solar Energy Materials \& Solar Cells, 94 (2010) 1686-1696. 
[28] D. Riveros-Rosas, M. Sánchez-González, C. A. Arancibia-Bulnes, C. A. Estrada, Influence of the size of facets on point focus solar concentrators, Renewable Energy 36 (2011) 966-970.

[29] J. A. Duffie, W. A. Beckman, J. Mcgowan, Solar Engineering of Thermal Processes. Wiley (1980).

[30] S. Kalogirou, Parabolic trough collector system for low temperature steam generation: design and performance characteristics, Applied Energy 55 (1996) 1-19.

[31] C. F. Chen, C. H. Lin, H. T. Jan, Y. L. Yang, Design of a solar concentrator combining paraboloidal and hyperbolic mirrors using ray tracing method, Optics Communications 282 (2009) 360-366.

[32] H. M. Guven, R. B. Bannerot, F. Mistree, Determination of error tolerances for optical design of parabolic troughs. Am. Soc. Mech. Eng. (Pap.) (United States) (1983) 83-wa/sol-1.

[33] J. M. Gordon, D. Feuermann, Optical performance at the thermodynamic limit with tailored imaging designs, Applied Optics 44 (2005) 2327-31.

[34] TracePro User's Manual. Release 7.0 Chapters 5-6. Lambda Research Corporation. 25 Porter Road, Littleton, MA 01460, USA (2011). 


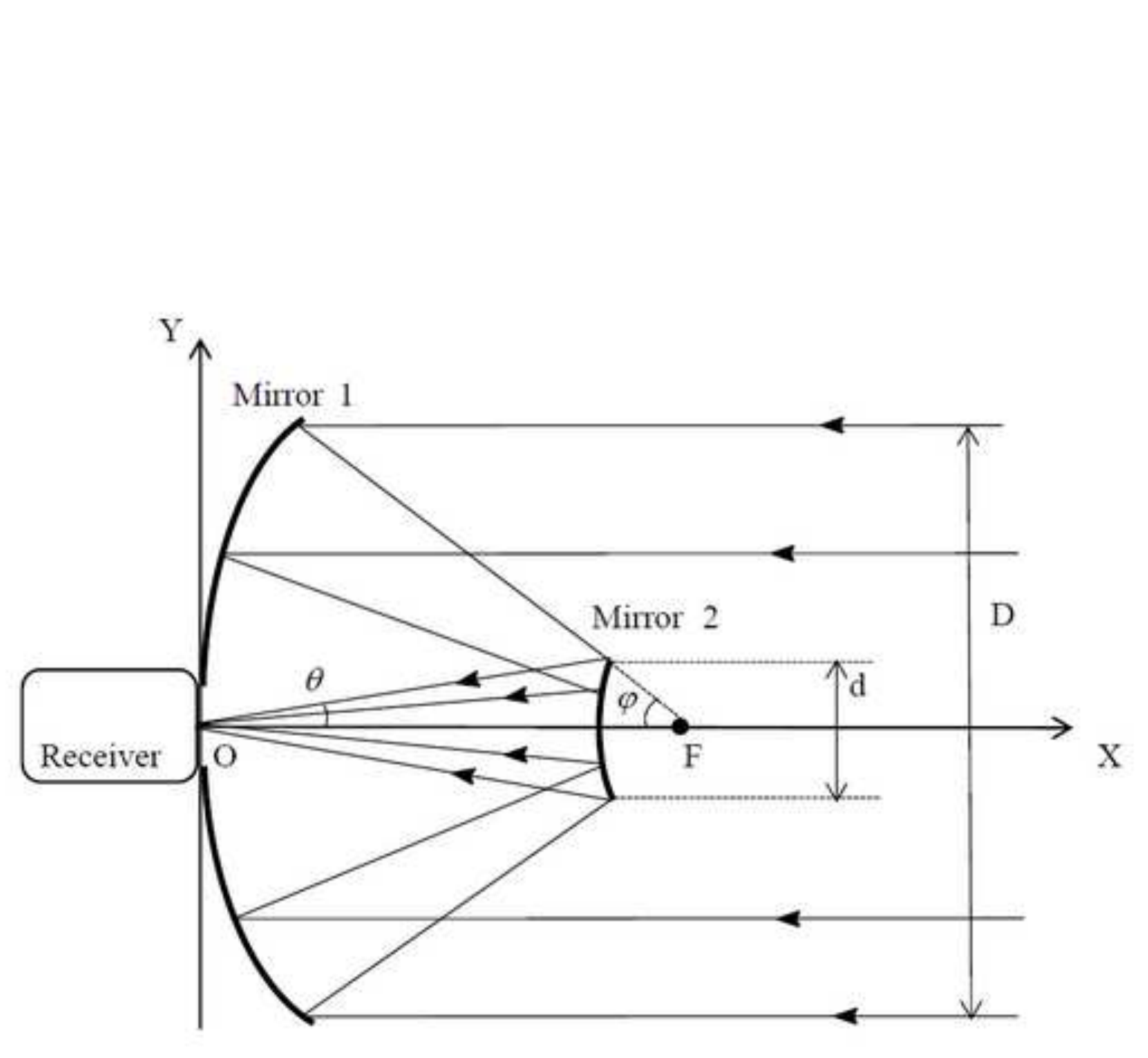

Figure 1

.

1

\section{政}

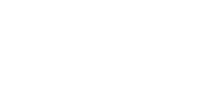

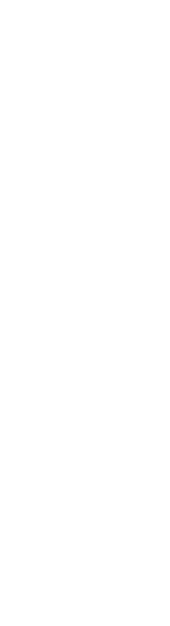

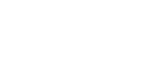

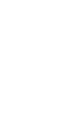




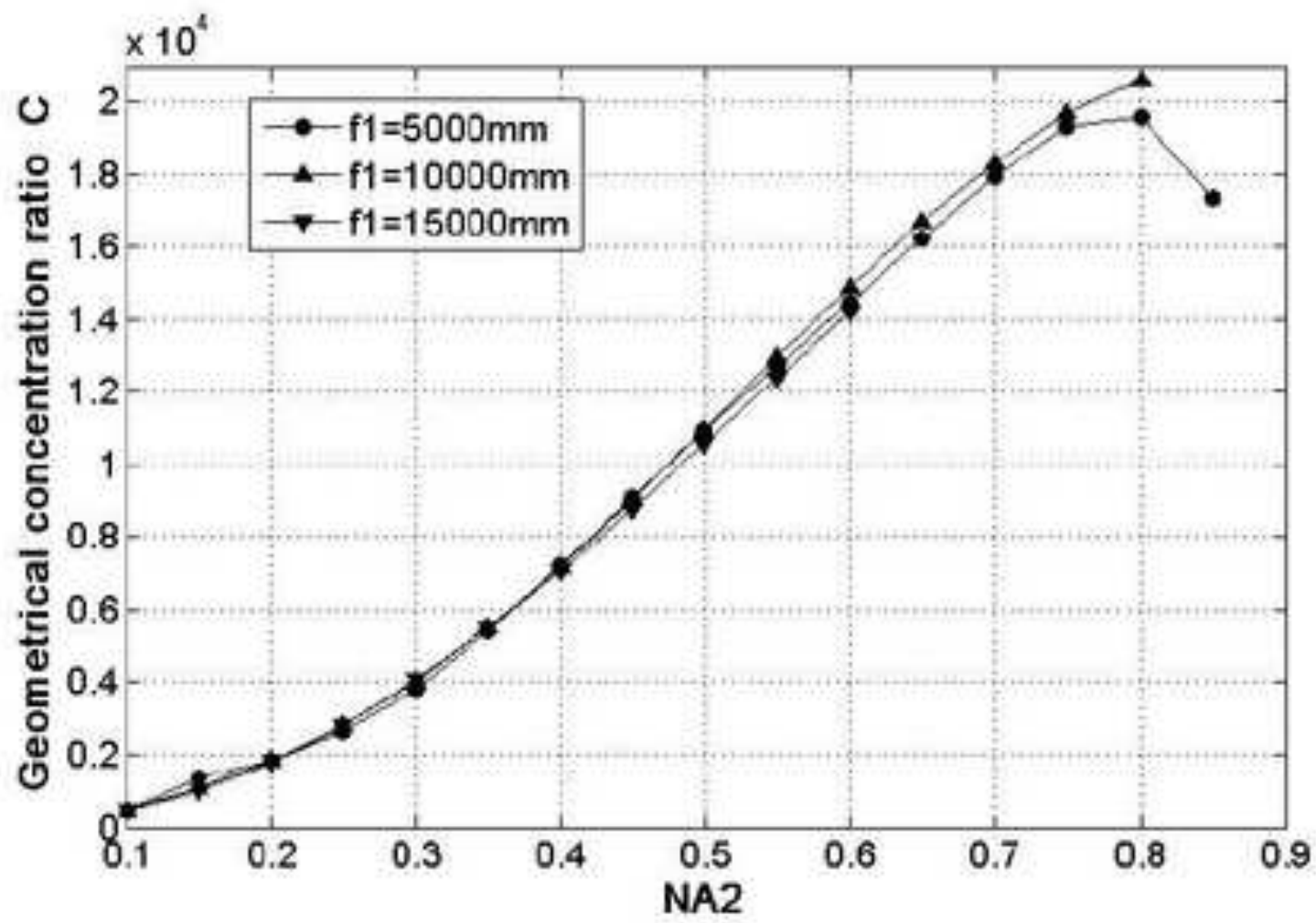

(a)

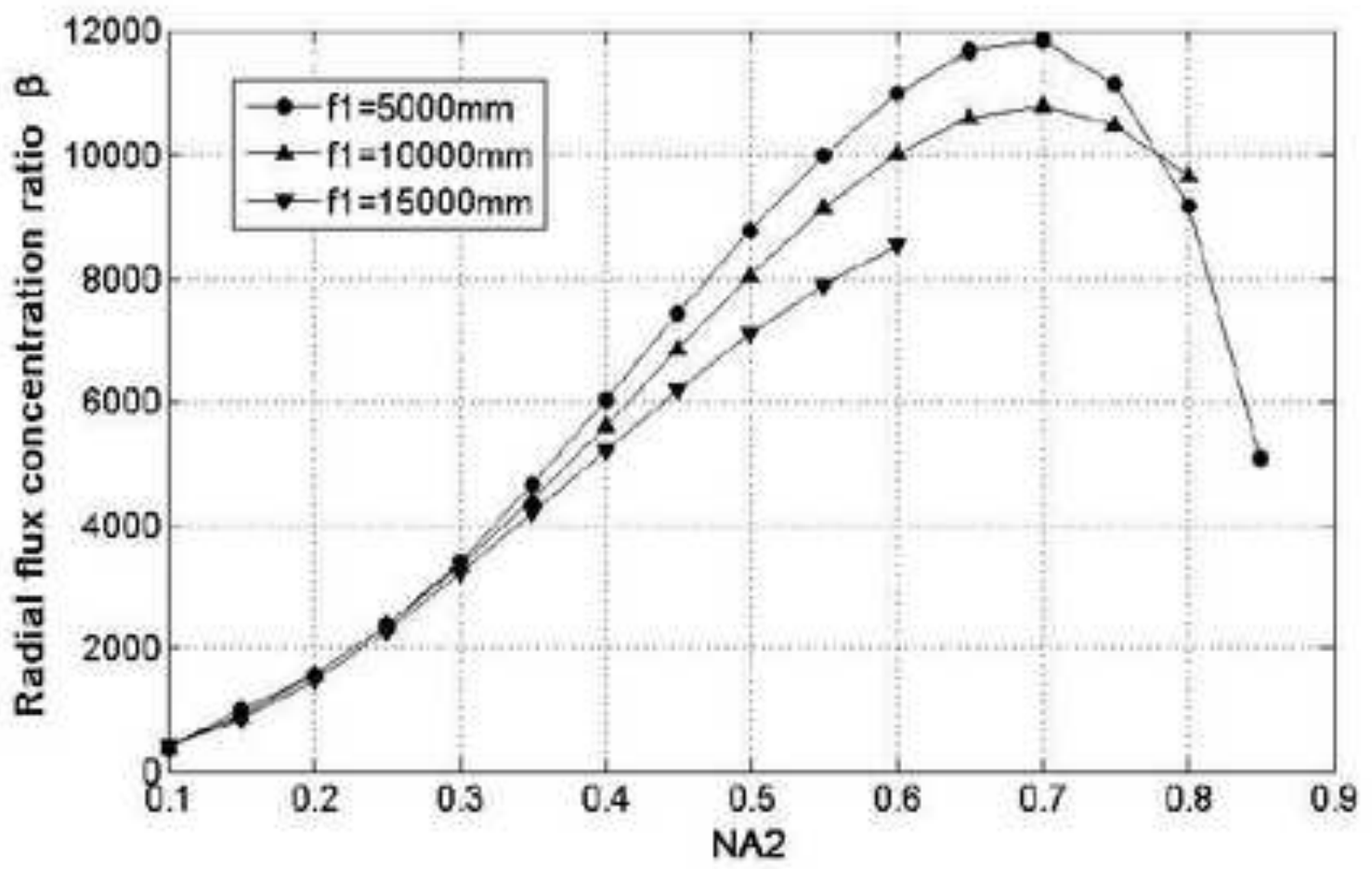

(b) 


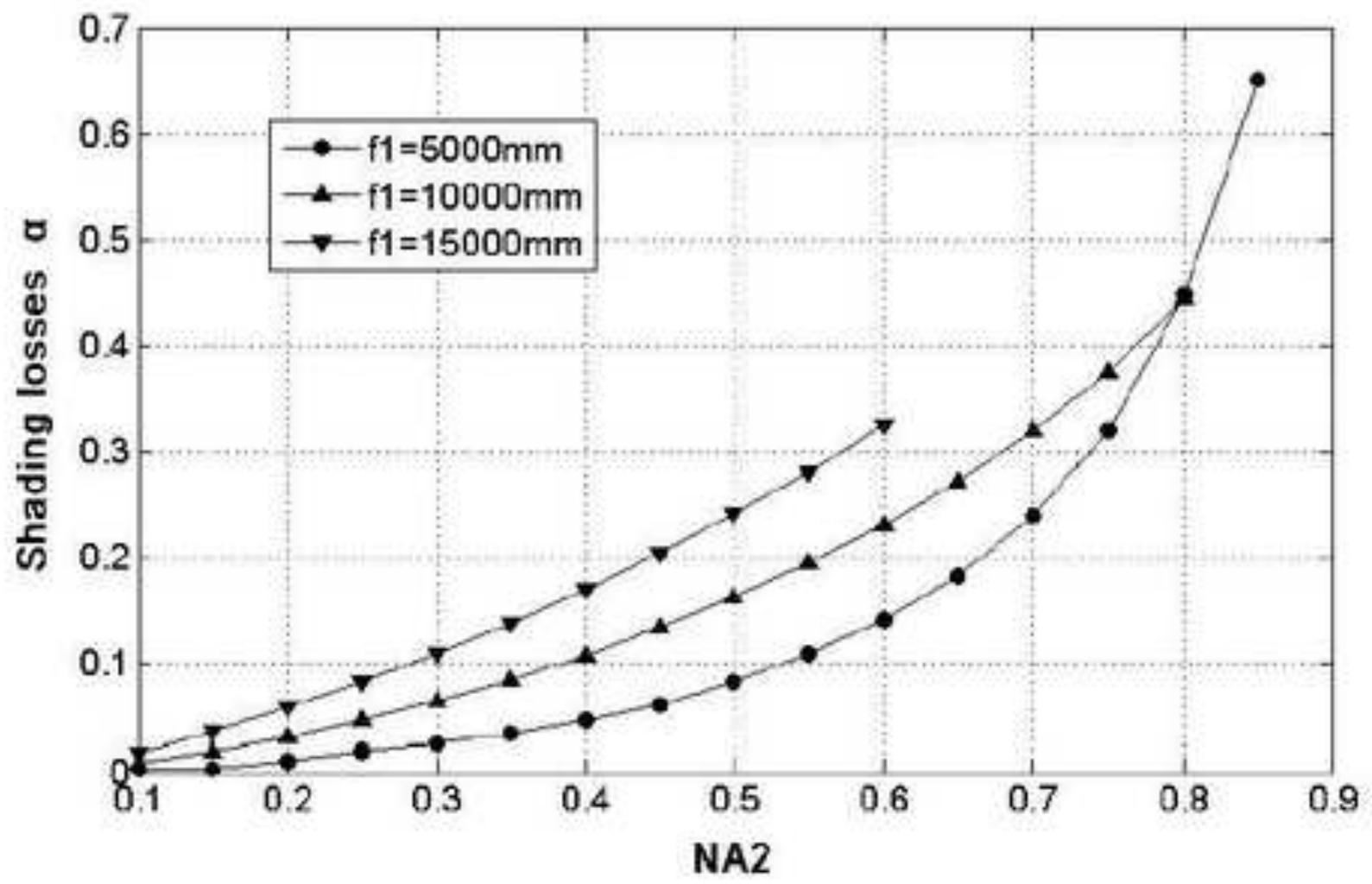

(a)

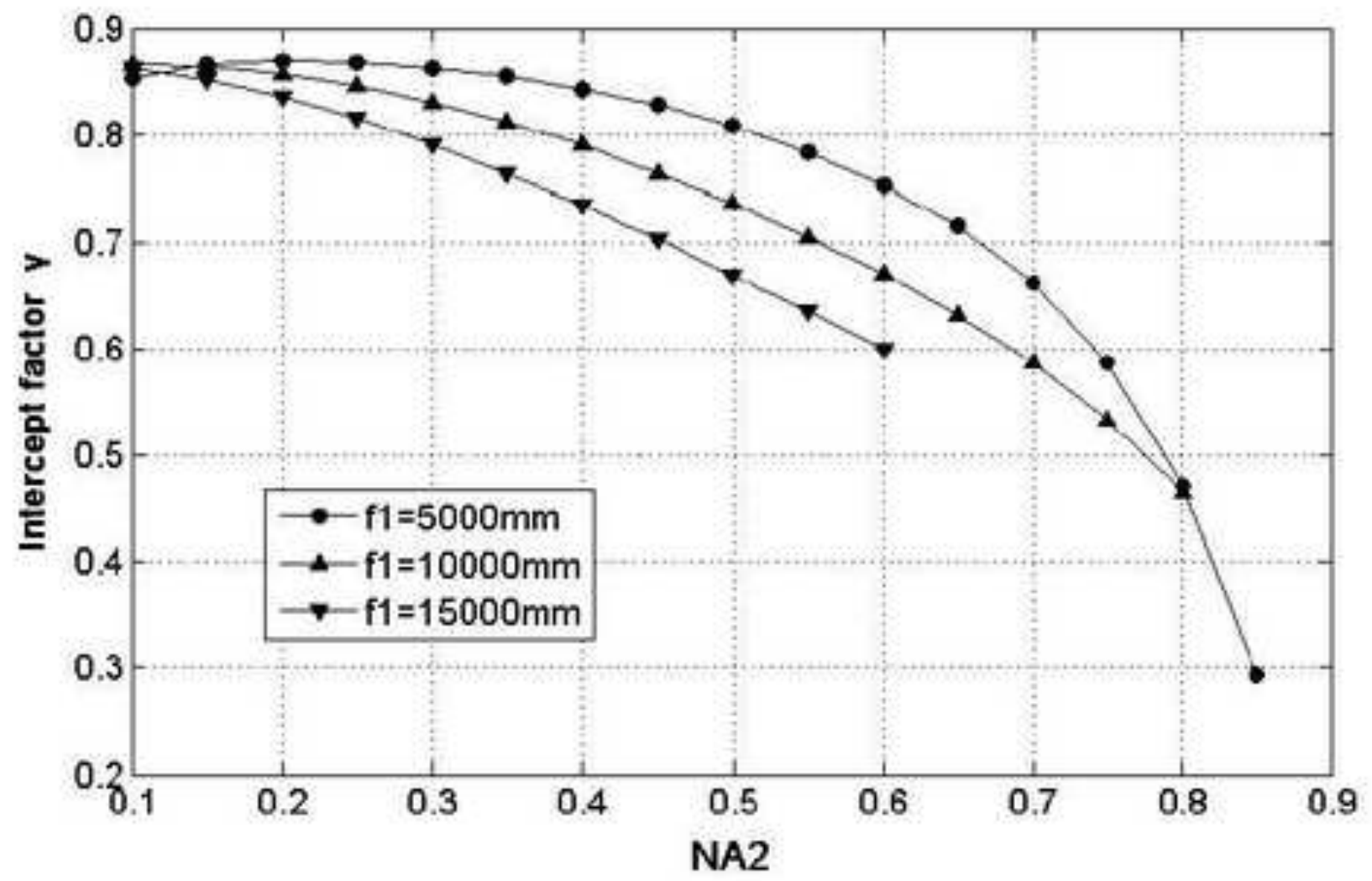

(b) 


\section{Figure 4a}
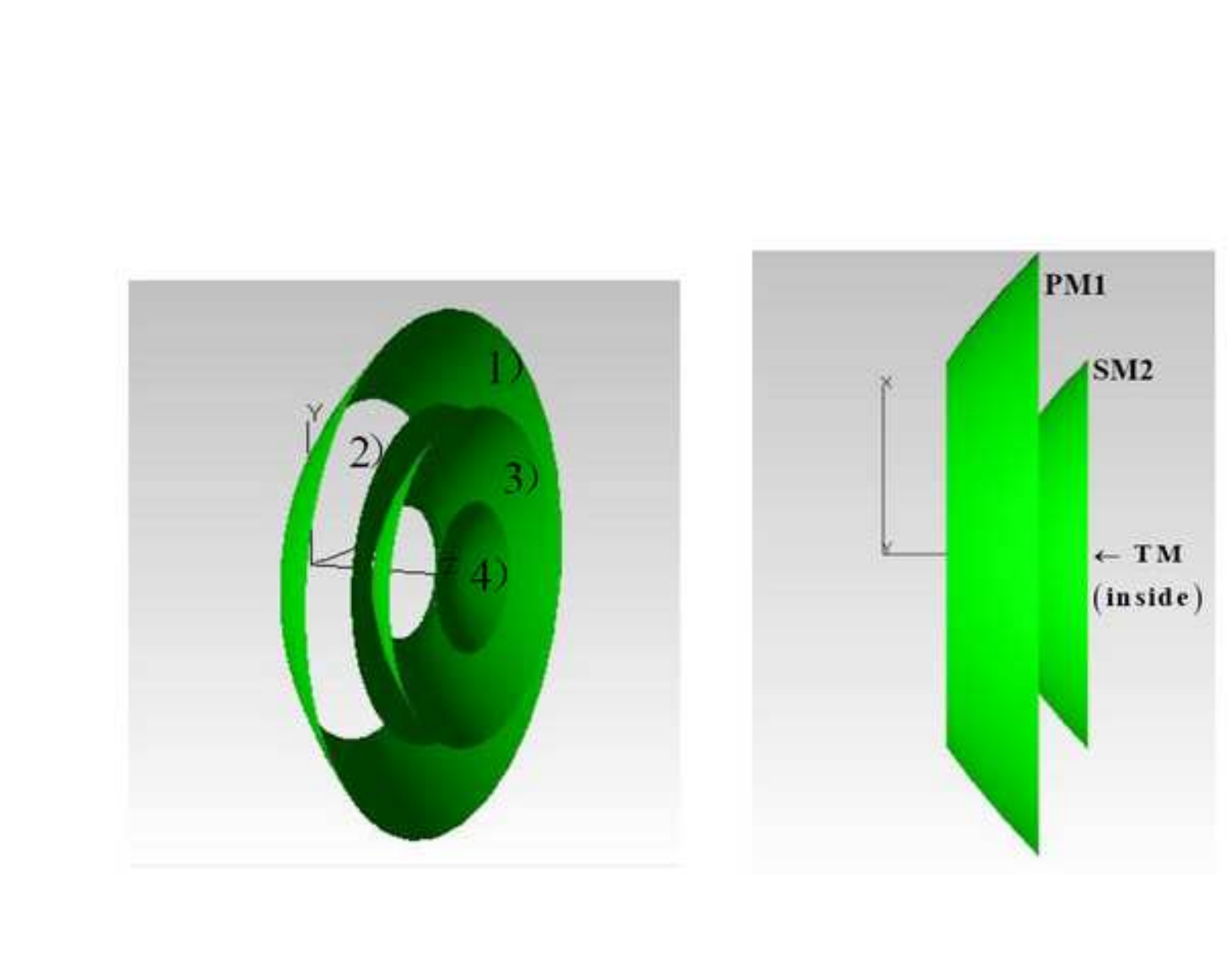

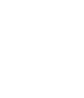
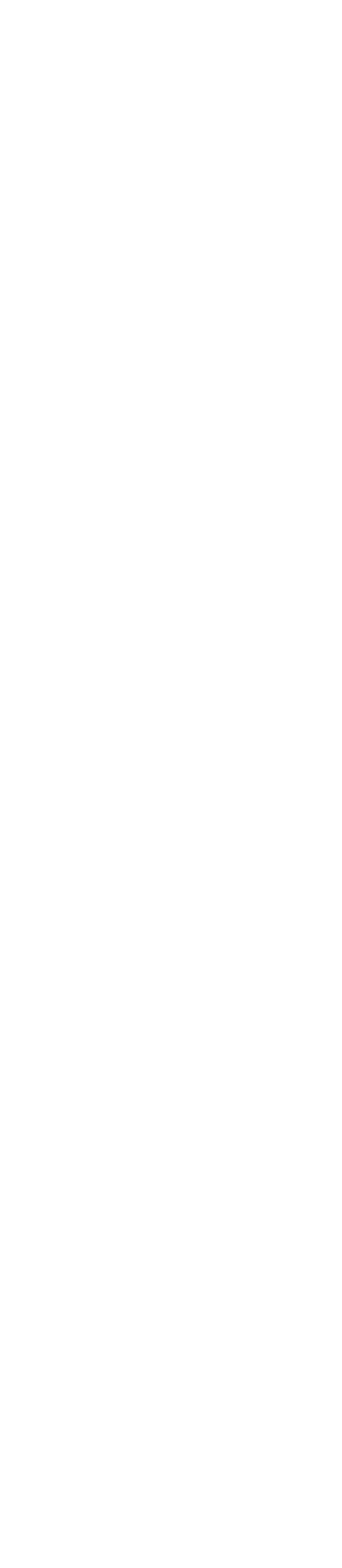
inside) 


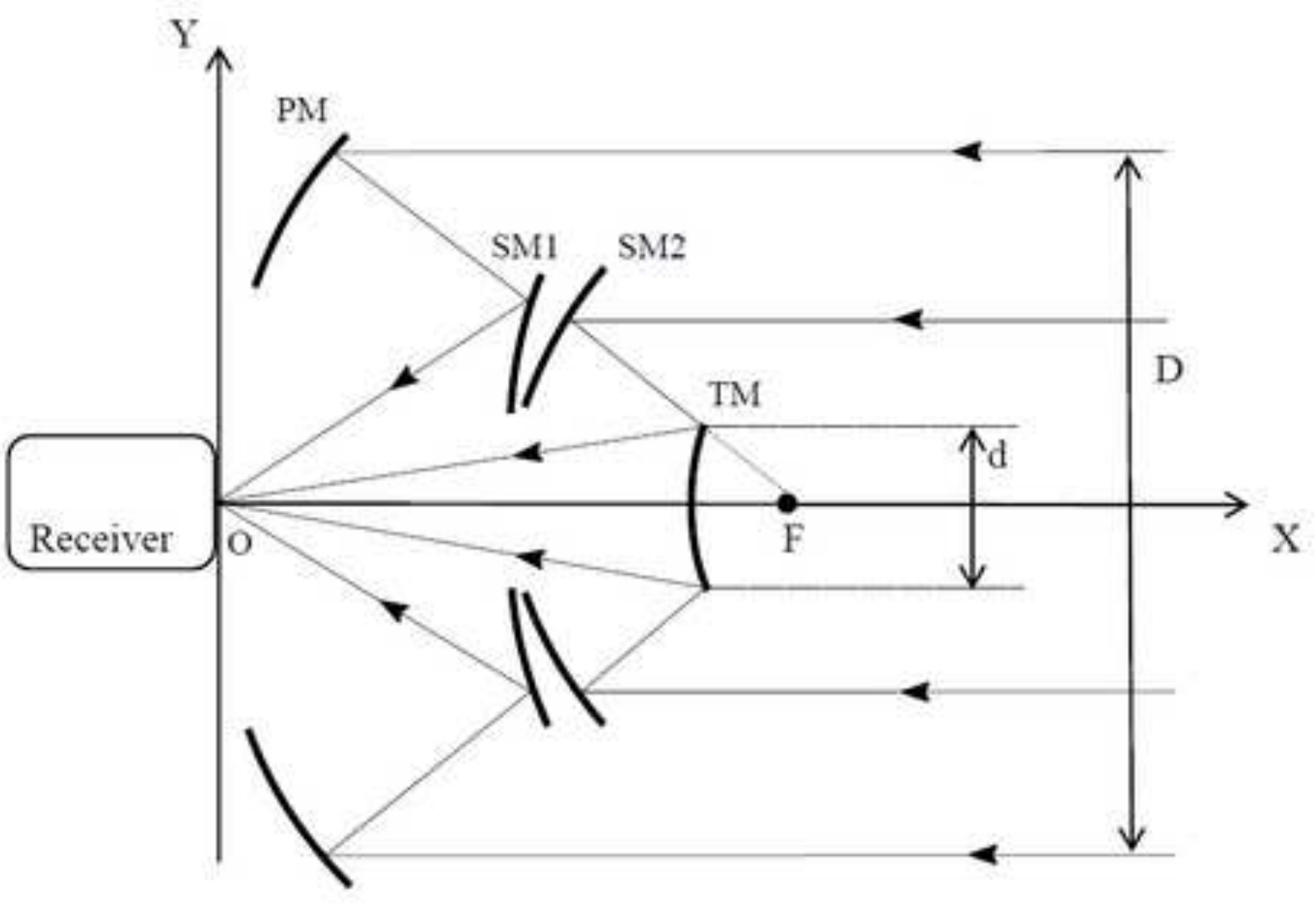


Figure 6

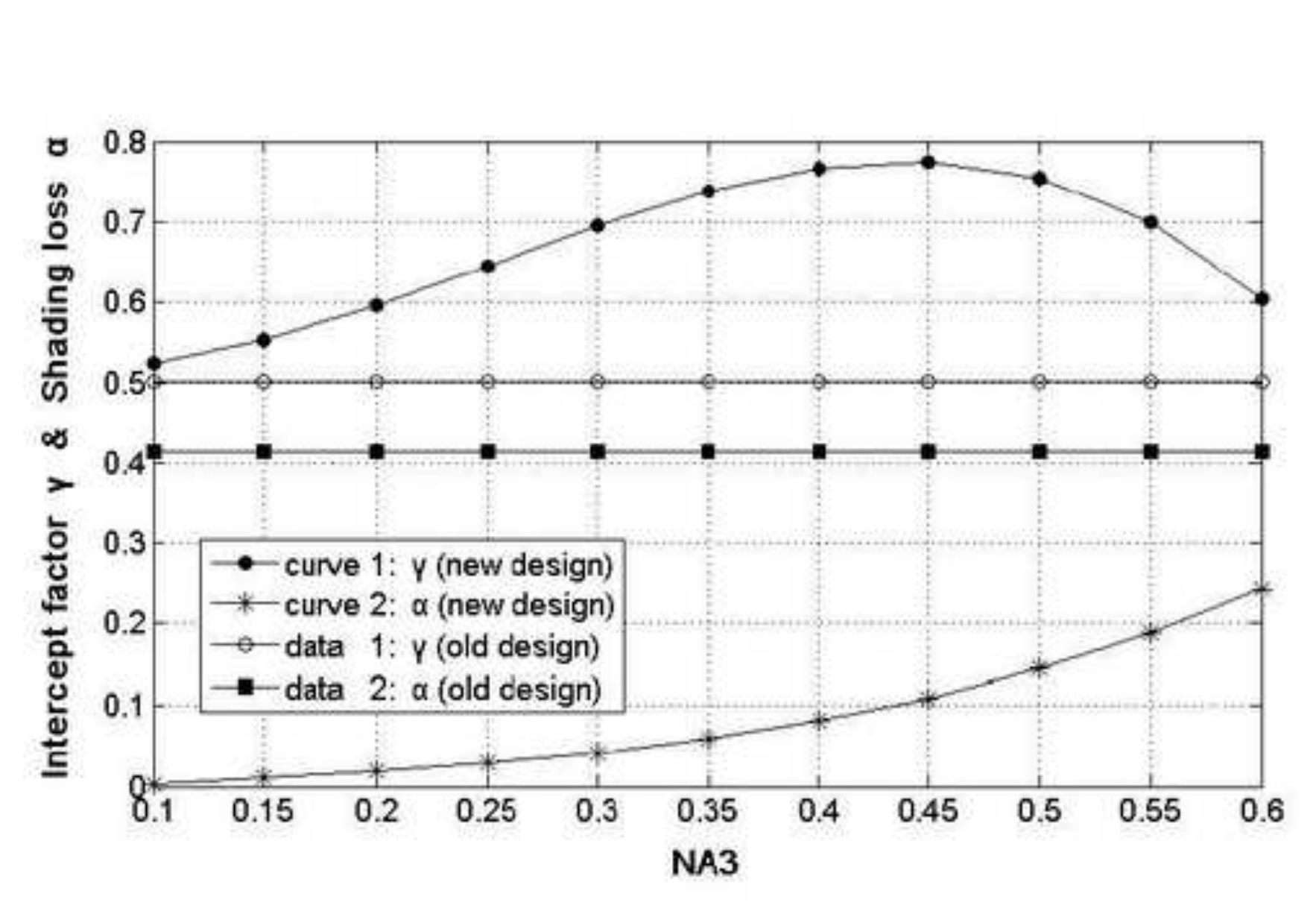

列

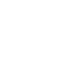

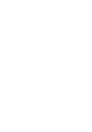

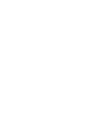


Figure 7
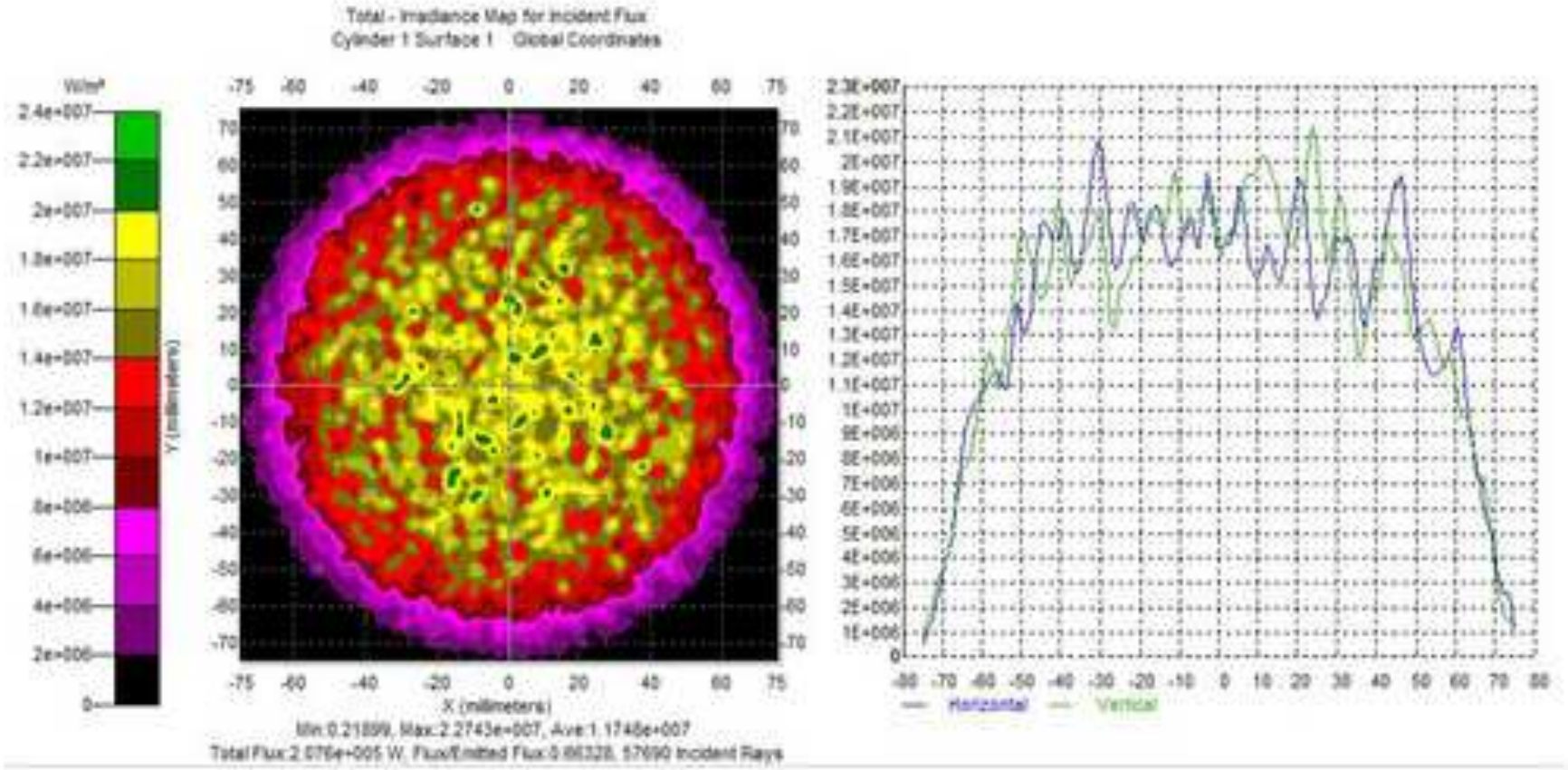

(a)
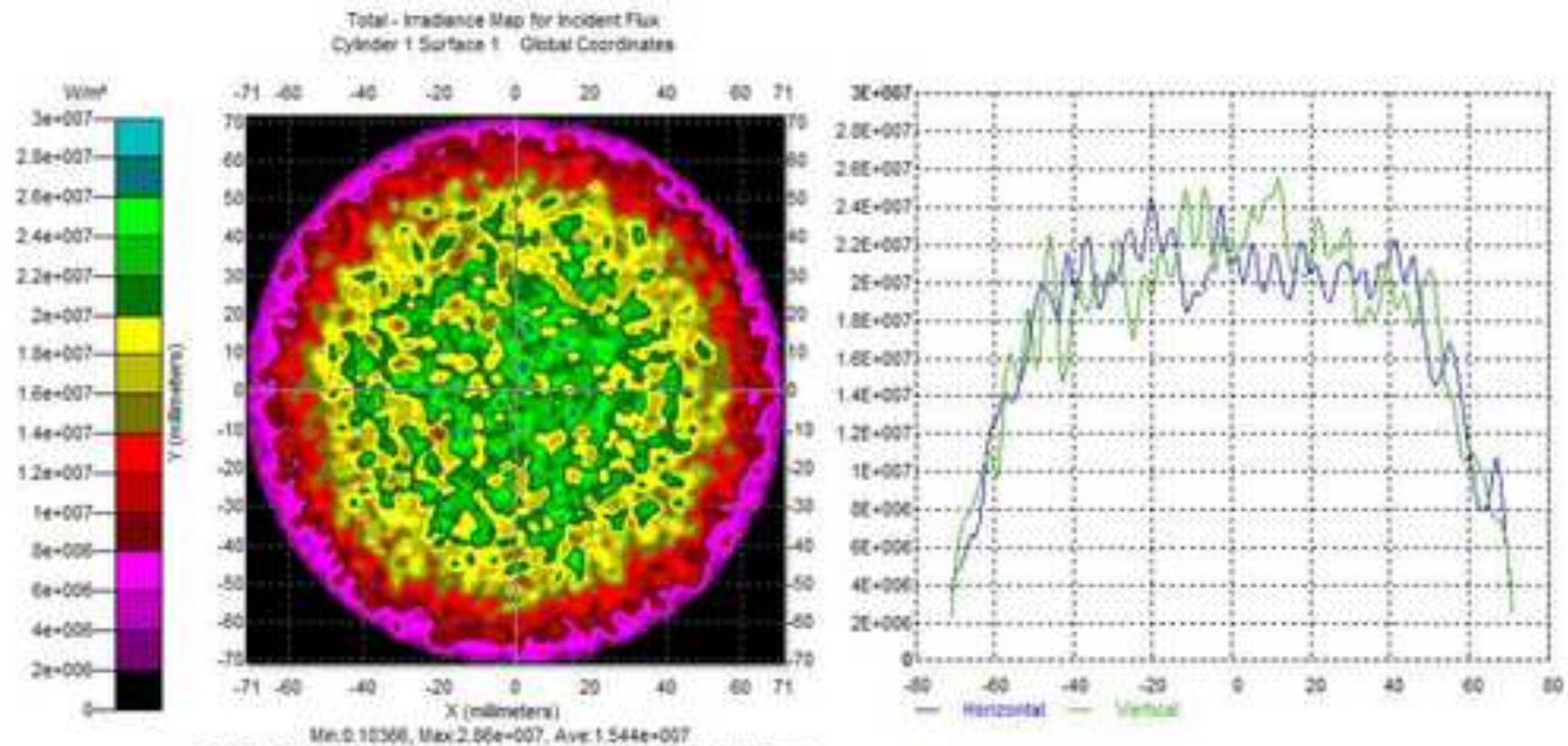

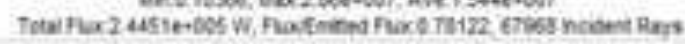

(b) 

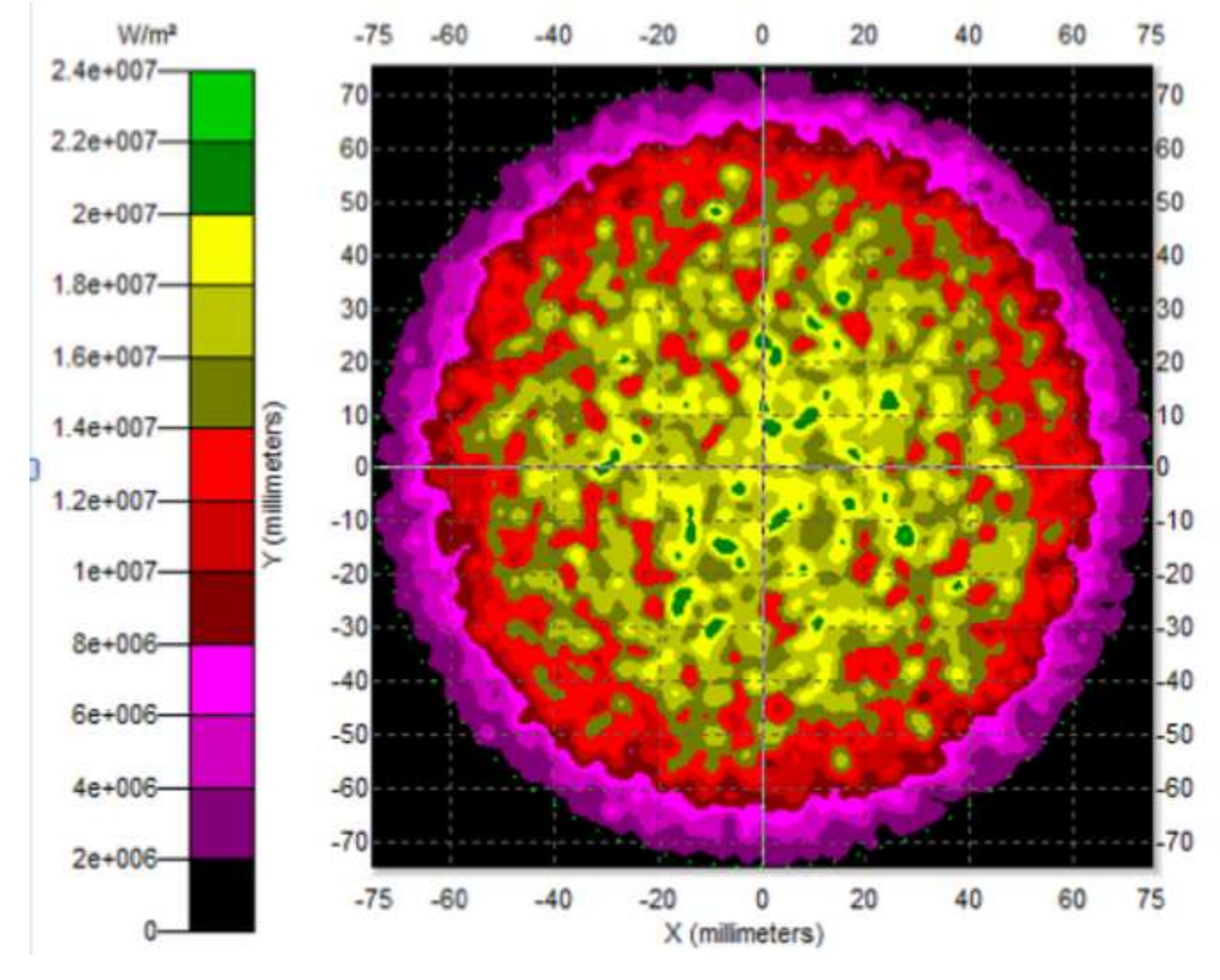

Graphical A 